\title{
Prognostic and health management design for subsea applications
}

\author{
Xiaojing Gao, Octavian Niculita, Don McGlinchey \& Babakalli Alkali \\ Department of Engineering and Built Environment, Glasgow Caledonian University, Glasgow, UK
}

\begin{abstract}
The design of a subsea production system needs to ensure high reliability and safety figures since these assets will be deployed in harsh environments for extended periods of time. Maintenance costs associated with these systems represent a significant percentage of the total operational expenditure incurred by an Oil \& Gas operator. Traditional reactive maintenance approaches applied on subsea equipment are starting to drop in their efficiency as degradation occurs on such systems resulting in prolonged downtime periods. Prognostics and Health Management is a relatively new topic and other industry sectors have demonstrated that it can provide a solution for reducing maintenance costs and improving systems' overall availability. This paper presents a prognostic and health management development process suitable for subsea production systems.
\end{abstract}

\section{INTRODUCTION}

When oil and gas exploration is not economically viable through tradition oil platforms, subsea production systems represent an alternative for the majority of operators. A Subsea Production System (SPS) is a collection of hydrocarbon extracting equipment located on the seabed and its main components consist of a seabed wellhead, subsea X-mas tree (XT), manifold, umbilical, riser, a network of pipelines, flowline as well as subsea power and control systems. The amount of shallow water oil and gas reserves is decreasing. This has led exploration and production into deep waters where SPS are the preferred solution to make development economically viable. The subsea industry is now facing a number of challenges on how to improve reliability and safety of critical assets in an economical way. Subsea lifecycle analysis demonstrated that Capital Expenditure and Reliability Availability Maintenance Expenditure (RAMEX) are the two major costs of a subsea asset, with downtime cost making up the majority of RAMEX. Prognostic and health management (PHM) has the capability to address these financial challenges and reduce the downtime cost by assessing and predicting the Remaining Useful Life (RUL) of a component/system while supporting the system's goal and compliance with high level requirements- safety, reliability, availability, maintainability etc.

At present, the main research focus area of PHM in the context of subsea applications is only targeting the development of such capability for isolated components. This paper introduces on an integrated approach to design the PHM for a SPS at the system level. This approach is mapped on a typical engineering design and operational process of a subsea system and it involves integration and concurrent analysis of multi-disciplinary sources of knowledge and interaction between several engineering functions.

Section 2 of this paper discusses the current prognostic approaches and the level of adoption of PHM for subsea equipment. Section 3 will cover four engineering disciplines and their potential use for the development of the PHM capability. Section 4 will present a novel PHM development process capable of integrating knowledge, information and data within concurrent engineering analysis. In section 5, an instantiation of the PHM development process for a XT will be presented.

\section{STATE OF THE ART OF PHM}

Four different types of prognostic approaches currently exist:

- Experience-based prognostic approaches are based on historical data and knowledge accumulated during the lifecycle of systems.

- Model-based approaches involve the construction of mathematical model which integrates the underlying physics of failure of the critical components of the system, their degradation and their failure modes.

- Data-driven prognostic approaches specify the behavior of a system through gathered operational data (CM data via sensors and/or event data). The data is processed and compared with key parameters/features to predict the probability of fault occurrence. 
- Hybrid prognostics are a combination of the model-based and data-driven with parameters in the model being continuously updated when data from service becomes available with the ultimate purpose of improving the accuracy of the prediction (Vachtsevanos, Lewis, Roemer, Hess, \& Wu, 2006) (Medjaher \& Zerhouni, 2013), (da Silva \& Radespiel, 2013).

The majority of industry sectors adopted the data-driven prognostic approaches as a first attempt for the development PHM capability, particularly for complex systems (Bykovsky, 2008) as this enables the development of the understanding of degradation without the construction of mathematical model capturing the physics of failure. Currently, in the subsea arena, there are very few Condition Based Maintenance (CBM) solutions deployed in field. The major Original Equipment Manufacturers (OEM) for SPS include companies like: Technip/FMC Technologies, Cameron, GE, Aker Solutions, OneSubsea etc. Based on information available in the public domain, Cameron provides a blowout preventer condition based monitoring system and riser annulus condition system. However, FMC has delivered a first attempt of a system level Condition and Performance Monitoring (CPM) capability for a subsea asset, currently being installed at the Gjøa field (Soosaipillai, Roald, Alfstad, Aas, Smith \& Bressand, 2013).

\section{ENGINEERING DISCIPLINES AND THE STAKEHOLDERS INVOLVED IN THE SUBSEA PHM DESIGN}

\subsection{Overview}

To develop and implement any of the prognostic approaches mentioned in the previous section, different types of data and information is required (Vachtsevanos, Lewis, Roemer, Hess, \& Wu, 2006). This information and data represents the output of several engineering disciplines being owned by various functional teams. The design stage for a subsea system is usually undertaken by the OEM and suppliers under the requirements established by an operator. During the operation period, maintenance teams will be hired by the operator to Inspect/Maintain/Repair (IMR) the subsea equipment, although, the maintenance of control systems will be the responsibility of the OEM. Very often, upgrades, overhauls and de-commissioning typically are done by different parties. During the life time of a subsea production system, multiple organizations are involved and a subsea system may include components and processes originating from all over the world. Throughout the entire lifecycle of a subsea field, interactions between different engineering disciplines belonging to different companies, which are operating under multiple languages and cultures, also take place. Hence, one of the challenges is the fact that the data/information required to develop a PHM solution is not unified and/or centralized. In the context of subsea applications, these engineering functions and the data/information associated to each of them is captured in Table 1.

\subsection{Subsea system design - initiation of the PHM design}

PHM Design must be underpinned by a level of understanding of the healthy state of a system. In the case of a subsea system, this characterization is developed during various stages of the design process (conceptual design, front end engineering design (FEED) and detailed design). Engineering modelling is carried out during the FEED. Modifications to the design (to include the PHM capability as an afterthought) might be extremely costly, therefore it is recommended to design-in the PHM function as the asset design progresses through various technical and business gates. It is instrumental at this stage to derive the PHM requirements from the subsea asset requirements, if such a capability is to be developed.

\subsection{Reliability and availability analysis- foundation of the PHM design}

In the offshore industry, Failure Modes and Effects Criticality Analysis (FMECA) tends to be one of most common approaches for reliability analysis and it has been increasingly implemented in the last ten years on subsea projects (DNV, 2013). FMECA also forms the foundation for good PHM design (Vachtsevanos, Lewis, Roemer, Hess, \& $\mathrm{Wu}, 2006)$ although, in the context of subsea

Table 1. Engineering disciplines.

\begin{tabular}{lc}
\hline Engineering disciplines & Information \\
\hline System design & Engineering models \\
& Schematics, Reports, \\
& Hierarchical levels \\
& Dependencies \\
Reliability \& & Failure concepts \\
Availability & Criticality information \\
& (Re) Certification \\
Control and & Operational conditions \\
Instrumentation & Condition, Performance \\
Condition and & Indicators Control data \\
Performance & \\
Monitoring (CPM) & \\
Inspection, & Past operational conditions \\
Maintenance and & Maintenance records \\
Repair (IMR) & Failure history \\
\hline
\end{tabular}


equipment, it is mainly used to support qualification of new technologies or re-qualification of legacy systems. Reliability analysis must be based on the actual design of the system therefore a concurrent engineer design and reliability analysis is recommended to ensure the both disciplines are targeting the same point of truth. Input is required from IMR to ensure the efficiency and accuracy of this analysis. A FMECA study attempts a good understanding of system behavior under faulty conditions is instrumental to be able to design the diagnostic and prognostic capability. Historical data and knowledge can also be employed to adjust or re-design components/equipment/systems to improve reliability targets. This information usually resides with the operators and OEMs, but it is very often not available for the reliability team responsible with the qualification of the subsea equipment under investigation.

\subsection{Control \& instrumentation/CPM-core of the PHM design}

A large number of parameters to assess the condition of an SPS is currently captured by CPM through sensors i.e. acoustic control systems, multiphase flow meters, accelerometers, pressure and temperature sensors, sand and leak detection systems, as well as detectors for dropped objects (ISO:13828, 2010). These types of sensors found their way into the subsea design either through regulations or recommendation and there is a consensus in the industry that they are not engineered for the purposes of ensuring higher availability figures. Production efficiency performance of subsea equipment has been particularly poor in recent years, reaching a low point of $60 \%$ in 2012 and averaging at $71 \%$ in 2015 although back in 2004 the production figures were above $80 \%$. Degradation of production equipment is one of the contributor factors to this drop. Abnormal behavior of subsea equipment can be detected by condition monitoring solutions and information sensed by instrumentation is sent to the control module and interpreted by the operational teams (Markeset, Moreno-Trejo, \& Kumar, 2013) to allow informed decisions guiding the operation and maintenance.

\subsection{Maintenance - exploitation of the PHM design}

Various types of maintenance strategies exist and can be implemented for subsea equipment in service. Corrective maintenance is typically applied after the failure has occurred. A scheduled maintenance regime aims at dealing with faults before they occur, but they are based on fixed time intervals. The conditional maintenance only supports non-dynamic estimation of the degradation and the most recent approaches take advantage of the PHM capability to estimate the RUL of a critical component and to plan the maintenance job according to these calculations (based on data-driven, model-based and hybrid prognostics algorithms). The PHM capability of a system is represented by a set of techniques and methods from different disciplines that combine knowledge and data to support predictive maintenance by detecting, diagnosing, predicting, advising and analyzing (postmortem) the failure information (Guillén, Crespo, Macchi, \& Gómez, 2016). The information and data related to functional failures (failure modes) and physical failures (faults) of subsea equipment is scarce, so the traditional reactive maintenance approaches (corrective and scheduled) are still the preferred choices for oil and gas operators. The predictive maintenance regimes also present their own set of challenges and these must be considered during the design stage (the inability to accurately and reliably predict the RUL of a component/system; the inability of maintenance systems to document, learn and recommend that action should be taken; the lack of tools capable of demonstrating the effectiveness of a predictive maintenance program). SPS have been typically designed to operate over five years without failure, thus the operator will plan to carry out preventive maintenance every five years (Moreno-Trejo \& Markeset, 2012). However, over the last decade, reliability data shows some components had to be maintained/replaced sooner to prevent failure. Hence, traditional maintenance can incur huge expense and consequential damage for an asset and the environment - such as pollution, loss of production, etc. (Markeset, Moreno-Trejo, \& Kumar, 2013; Uyiomendo \& Markeset, 2015). In recent years, traditional maintenance activities in the oil and gas industry are transforming through the adoption of CBM which are ensuring efficient maintenance, reducing lifecycle costs and improving the systems' overall availability. The application of CBM and PHM (as an extension of CBM) in the oil and gas industry has started to be exploited on for example, drilling systems, control systems and pumping systems.

\section{SUBSEA PHM DEVELOPMENT PROCESS}

To determine the Prognostic method, these four major disciplines need to share information and data with each other. However, PHM development for an entire subsea system is still a challenge from the view of big data management and information support, thus it is hard to build those disciplines into system.

In this paper, we propose an integrated approach to subsea PHM design process. This process is captured in Figure 1 and it highlights the data/ information exchanged between the four main engineering disciplines, discussed in the previous 


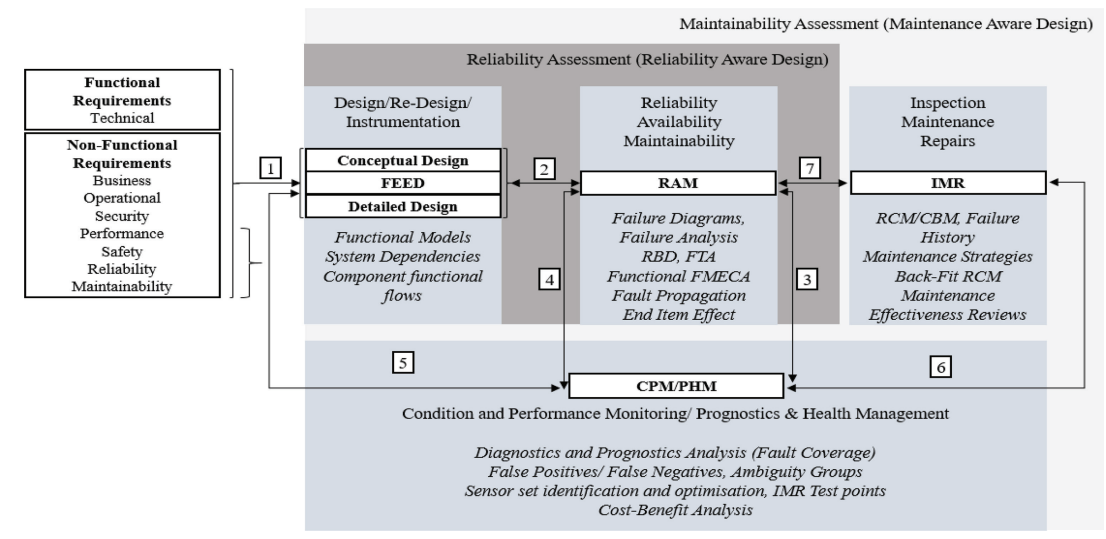

Figure 1. An integrated approach for exchanging engineering knowledge to support Subsea PHM Design.

section. The proposed subsea PHM development process includes feedback loops that are intended to enable enhanced data collection, exchange and analysis of knowledge related to the degradation of subsea components, addressed by different engineering disciplines as the subsea project moves through various technical and business reviews. Good communication during a generic design engineering process includes both historical and current information to be shared freely, problems to be reported, views to be exchanged, and positive interpersonal relationships to be retained, as the design progresses through various technical and business gates. However, the effective communication is also dependent on balancing the levels of information to the complexity of the task/topic, avoiding both over-complication and over-simplification. Hence, suitable levels of information must be delivered to the correct person at the correct time to communicate essentials without the receiver being overburdened with data (Parkes \& Hodkiewicz, 2011).

Step 1 - The start of the PHM design process is represented by the requirements phase. HighLevel (HL) requirements are typically divided into functional requirements (FR) and non-functional requirement (NFR) categories. Functional requirements define the technical details of a system (including the function of the system and the functions of each individual component) and non-functional requirements cover the attributes of the system (such as safety, reliability, maintainability, usability, performance, security, etc.). The Design/Re-design/ Instrumentation phase of the PHM development process coordinates various engineering analysis to ensure the final subsea design is meeting the requirements established at the start of the project. During the design phase, data associated with the environmental conditions, reservoir, well completion, process and operations, host facilities, safety and hazards should be considered when progressing through conceptual, FEED and detailed design. These engineering efforts are targeting the technical requirements (also known as functional requirements (FR)) of a subsea system since this design element is heavily regulated and supported through recommended practices (ISO 13628-1, 2010). NonFunctional Requirements are also considered by the current subsea design best practices and they cover safety, performance and security. However, there is no attempt to define and to target the reliability and maintainability requirements at the early stage of the design. This influences the PHM requirements definition as these are derived from the reliability and maintainability requirements. Only recently, the industry has generated recommended practices like the API-RP-17 N on topics related to reliability, technical risk and integrity management (API RP $17 \mathrm{~N}, 2009$ ). However, they are not yet adopted due to the lack of tools, processes and meaningful reliability data to support their implementation. Reliability and maintainability requirements should be part of the non-functional requirement (NFR) of the system. Deriving PHM requirements should be done from system's high-level (HL) Non-Functional requirements. For example, a HL NF requirement for a XT can be affordability by reducing the downtime periods while keeping the same levels of safety. In this manner, cost and safety become main drivers for the development of a PHM solution. Derived PHM requirements can be represented: PHM Requirement 1 - the XT must have a feature that can reliably predict functional failures at least one week prior to the actual event and PHM Requirement 2: the XT must have the capability of offering mitigation/advisory generation in the context of current operational conditions. Having access to information provided by such features, the operator reasonable time to schedule a vessel, equipment, personnel to carry out an intervention on the faulty components. 
Step 2 - To support the PHM within the subsea design phase, reliability analysis needs to be carried out concurrently with the subsea system design. Reliability analysis should be based on the actual design data to support the engineering analysis that can identify the means to detect and isolate the potential faults which may occur during the operation. One way to support the realization of this step is through the exploitation of functional models which can support reliability engineers to carry out analysis such as FMECA, Fault Tree analysis (FTA), and Reliability Block Diagrams (RBD) from the very early stages of the subsea design process. These methods and techniques can also underpin the Reliability Centered Maintenance (RCM) and Back-Fit RCM analysis at later stages of the lifecycle. RCM includes four elements that are critical to a maintenance program aimed at improving availability of a given asset. These elements are: preservation of the system function, identification of the failure modes that can lead to functional failures (and sequentially downtime), prioritization of failure mode candidates based on Occurrence (O), Severity (S) and Detectability (D) by highlighting the Risk Priority Number (RPN =O $\times \mathrm{S} \times \mathrm{D}$ ) of each of the candidates and finally selections of applicable and effective tasks to control the failure modes. Back-Fit RCM builds on the same RCM principles by incorporated operational reliability figures (by updating the $\mathrm{O}, \mathrm{S}, \mathrm{D}$ parameters) and evaluating the applicability and effectiveness of the control measures. We believe that the reliability, availability and maintainability analyses should be the foundation of the PHM design as it can highlight the risk associated with a brand new subsea design or a legacy system using information from service (provided to the reliability team during Step 7 using the output of a bespoke maintenance analytics engines). Sequentially, using this information, the design will be assessed by the reliability authority during a technical review (targeted reliability assessment) and if the design fails to meet a specific target, the risk and the critical components must be addressed either through re-design, redundancy or addition of instrumentation. Informed trade-off studies between these three approaches must be in place to guarantee the final design meets all the requirements of the project. However, this topic is beyond the scope of this paper. Nevertheless, for far too long, reliability assessment on subsea equipment was carried out only to present a measured reliability figure to support re-certification of production equipment already in exploitation.

If the PHM is channeled as derived requirement from the RAM requirements, specific levels of targeted reliability can be achieved. Also, different PHM requirements and implementation strategies can be evaluated at this stage against specific sets of RAM requirements. Very often, in subsea applications, redundancy seems to be the option preferred by the system designers as this guarantees improved availability figures when the primary component/sub-system fails. The major drawback of the redundancy approach is that fact that it does not offer any indication of the RUL for the critical component, sub-system or system, therefore, our case for addition of instrumentation supporting the PHM capability. If instrumentation is required for diagnostics and prognostics purposes, this must be defined by the PHM analysts in collaboration with the subsea design and reliability teams. We believe that majority of the condition monitoring applications existent in a subsea environment were driven by vendors of sensors capable of targeting symptoms associated with specific failures. This approach captures failures in isolation and does not account for propagation of faults leading to functional failures of other components and sequentially to failure of the system. This limitation can be overcome using functional models of the system, functional relationships and failure/ effects dependencies in a system for both functional and physical failures, defined using widely accepted, well defined failure taxonomies. Once the stakeholders of the asset have validated the propagation tables (component's reaction to functional failures of the system) generated against analysis capturing various end-item effects. The end-item effect is the consequence a failure mode has on the operation, functional output of the system at the highest indenture level (an item's position in the system hierarchy relative to the top-level item).

What we propose in step 2 is an implementation of the API $17 \mathrm{~N}$ recommended practices through a concurrent design-reliability analysis by evaluating the actual reliability of the design by highlighting the effects of failure modes leading to functional failures.

Step 3 - enables an informed dialog between RAM engineers and PHM analysts by allowing the use of system level propagation table (a collection of all the failure mode signatures - the effects (throughout the system, and not just at the point of occurrence) of a given failure mode universe. Currently, the job description of a PHM analyst falls somehow under the control team although we believe that its responsibility and involvement goes beyond the control systems. The PHM team should liaise very closely with the design team as it aims at the identification and optimization of sensor set solutions capable of detecting, isolating and making predictions of a given set of failure modes considered under the PHM analysis. The mean of realization of this dialog is represented by the reliability models populated with failure and criticality information of failure concepts. The automated PHM instrumentation analysis aims to identify and optimize, in a systematic manner, the sensor set configurations capable of supporting the detection, diagnosis, prediction functions of a subsea asset to further enable advisory 
generation. It also aims to calculate, for each sensor set solution identified during this process, the fault detection and isolation characteristic representing the proportion of failure modes selected for PHM analysis that can be detected and identified by a given sensor set under consideration. The PHM instrumentation analysis must be able to allow modification of existing sensor arrangements based on user knowledge or trade-offs. Legacy sensors present on the system can be considered as part of this analysis, although qualification institutions and regulators will not easily accept interrogation of sensors used for fail-safe control purposes (functional safety sensors). During this step, criticality of failures affecting a subsea system must be considered during the PHM instrumentation analysis. For new subsea designs, no measured criticality information exists and this must be defined using input from various stake-holders (subsea designers, RAM team, Operators, IMR personnel) by taking into account qualification of new technology standards and recommended practices (DNV-RP-A203, 2011; DNV-DSS-401, 2012). For subsea legacy systems, the criticality should be considered given what failed in service and it can be characterized through occurrence, severity and detectability parameters by calculating a risk priority number for every single component of the subsea equipment. The PHM instrumentation analysis should be capable of running the identification and optimization algorithms for specific groups of components by focusing on specific targeted criticality. There is also a feedback loop between the PHM and RAM functions meant to ensure that the selected sensor set solution meets the reliability criteria of the system (as a sensor that will fail in service ahead of the component that it is monitoring for failure does not ensure higher levels of availability for the asset). This feedback loop is represented within the PHM development process by Step 4 .

Step 5 of the PHM development process enables the dialogue between the PHM analysts and the subsea design team. Although represented as a separate link/step, this dialog should take place concurrently with the reliability analysis of the instrumented subsea system. Decisions regarding unfeasible sensor set solutions are taken, and trade-off studies related to cost, weight, coverage, location, reliability, probability of detection, probability of prediction, likelihood positives and negatives ratios, physical constraints, loads, environmental conditions should be carried out by a multi-disciplinary team led by the RAM function. During this step, the reliability and maintainability requirements are verified and validated by group of experts. As a mean of realization of this dialogue, we propose a model-based approach as this will allow rapid generation of sensor sets spanning multiple levels of hierarchy for a subsea system by considering technical and economical metrics.
Step 6 facilitates the exploitation of the data provided by a PHM-enabled subsea system and the support offered to the IMR function. Data from sensors will be plugged to diagnostic and prognostic engines capable of supporting the IMR function on fault detection, fault isolation and ideally, prediction of the remaining useful life.

Step 7 of the PHM development process facilitates an implementation of an integrated analysisdrive sustainment activity. It provides traceability of the subsea maintenance activities when using PHM information. We recommend the use of function-based reliability models to gather, share and analyze maintenance data in order to enable automated failure and data reporting, analysis and corrective action system (FRACAS/DRACAS).

\section{CASE STUDY}

For the implementation of the integrated PHM development process for subsea equipment, a commercial-of-the-shelf software tool, namely Maintenance Aware Design environment $\left(\mathrm{MADe}^{\mathrm{TM}}\right)$ developed by PHM Technology was employed. It was used to carry out an instantiation of the process on a XT. The selection of this software package was based on the previous success in using it in aerospace industry sector on fuel system and environmental control systems (Hess, Frith \& Calvello, 2005). $\mathrm{MADe}^{\mathrm{TM}}$ is a 'model-based' engineering tool that can provide an integrated framework to manage, control and analyse the information and data throughout different disciplines including the design, safety, reliability, availability and maintainability for a high-value high-complex systems. However, a good inter-discipline communication requires expert document management and control, and in this instance this is achieved through a model gathering data and knowledge from various disciplined. The instantiation of the PHM design process was carried on a typical subsea XT. Several concurrent engineering analyses belonging to separate engineering disciplines, but derived from a single model of this XT (developed within $\mathrm{MADe}^{\mathrm{TM}}$ ) were carried out. The outcomes of some of these analyses are highlighted in Table 2 ( $a$ and $b$ ). These are briefly summarized further on. The functional model accommodates information describing the input and output flows of each component, the causal relationships between these flows that allow for systematic propagation of failures throughout the system and criticality data (retrieved from the 6th edition of the Offshore Reliability Data-Volume 2). Knowledge and data characterizing the failure of each of the component forming a subsea XT was added to the functional model using concepts defining the causes, mechanisms, faults, symptoms and the links to the functional failures previously 
Table 2a. Outcomes of implementation of the integrated PHM development process.

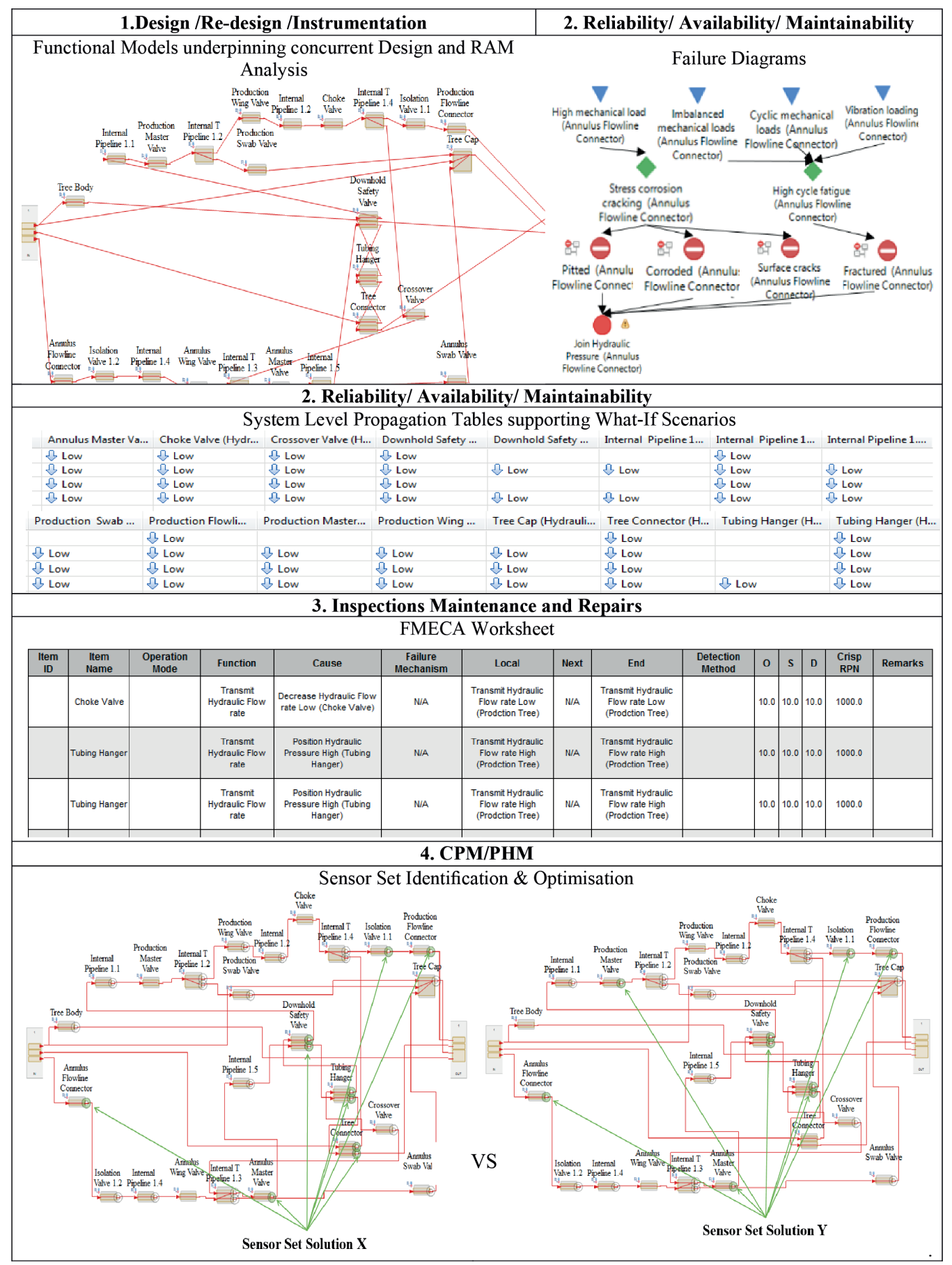

defined. Significant challenges were faced when trying to align the failure taxonomy used by the OREDA (based on the ISO: 14224 standard) and the failure taxonomy employed and defined by PHM Technology in MADe ${ }^{\mathrm{TM}}$.
A clear understanding of the causes, mechanisms, potential symptoms leading to a fault and the way this fault develops into a functional failure is instrumental in selecting the correct maintenance task or the PHM instrumentation, in an informed manner. 
Table 2b. Outcomes of implementation of the integrated PHM development process.

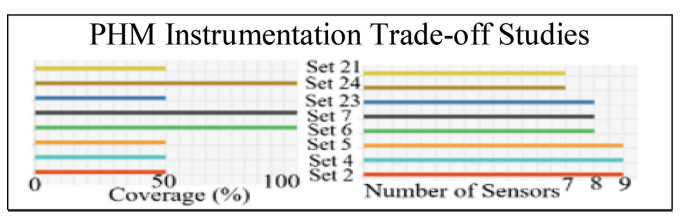

Four components were selected during the criticality assessment as having a significant impact on the function of the XT, namely the choke valve, the down hold safety valve, the production master valve and the tubing hanger. For the scenario of these four functional failures, 100 sensor set solutions were automatically generated from the functional model, having between 7-9 sensors (measuring pressure and flow rate) offering between $50-100 \%$ fault coverage. Ambiguity groups were clearly highlighted during this process due the similarities in the fault signatures characterizing two of the faults. At this stage, the PHM development process allow investigations on the trade-off studies on the sensor set solutions very early on during the design process and various maintenance strategies can be benchmarked when using specific diagnostic and prognostic engines coupled to the instrumentation identified in the previous step.

\section{CONCLUSIONS}

The definition and articulation of cost-effective maintenance regimes is a challenging task for subsea assets. Very often, they are over-engineered since they are required to operate in harsh conditions for long period of times. Various stakeholders are involved with these assets throughout the entire lifecycle of these assets and knowledge related with the de gradation of this equipment is scattered throughout various organizations being owned and used by different engineering functions. In this paper, an integrated PHM development process was presented as a multi-disciplinary engineering analysis, complementing the current development of subsea equipment. The proposed development process aims to help subsea designers to integrate the development of the PHM capability of a subsea asset with the actual design of such systems. This is meant to happen at the early stages of the design process, but the process also enables the retrofit of such capabilities on legacy subsea fields to achieve higher availability and operational reliability figures. This is achieved by placing the reliability, availability and maintainability engineering analysis at the heart of the subsea asset and PHM system level design.

\section{REFERENCES}

American Petroleum Institute (API). 2009. Recommended Practice for Subsea Production System Reliability and Technical Risk Management, API RP 17 N, 1st Edition.

Bykovsky, V. K. 2008. Data-driven modeling of complex systems. Berlin: Springer.

DNV-RP-A203. 2011. Qualification of New Technology.

DNV-DSS-401. 2012. Technology Qualification Management.

DNV. 2013. FMECA for Operational Planning of the Liwan 3-1 Subsea Development. Singapore: DNV.

Goldsmith, R. \& Ericson, R. 2003. Lifetime Cost of Subsea Production Systems (JIP). Norway: DNV.

Guillén, J. A., Crespo, A., Macchi, M. \& Gómez, J. 2016. On the role of Prognostics and Health Management in advanced maintenance systems. Production Planning \& Control, 27(12), 991-1004.

Hess A., Frith P. \& Calvello G. 2005. Challenges, Issues, and Lessons Learned Chasing the Big 'P': Real Prognostics Part 1, IEEE Aerospace Conference.

ISO 13374-4. 2015. Condition monitoring and diagnostics of machine communication and presentation.

ISO 13628-1. 2010. Petroleum and natural gas industries - Design and operation of subsea production systems - Part1: General requirements and recommendations.

ISO 14224. 2016. Petroleum, petrochemical and natural gas industries - Collection and exchange of reliability and maintenance data for equipment.

Ivanova, M. \& Brkic, I. 2015. Global Offshore Prospects and the Future of the North Sea. London: Subsea Integrity and Efficiency Conference.

Markeset, T., Moreno-Trejo, J. \& Kumar, R. 2013. Maintenance of subsea petroleum production systems: a case study. Quality in Maintenance Engineering 19(2), $128-143$

Medjaher, K. \& Zerhouni, N., 2013. Hybrid prognostic method applied to mechatronics systems. Advanced Manufacturing 69(1-4): 823-834.

OREDA. 2015. OREDA Offshore Reliability Data. Høvik: OREDA Participants.

Parkes, K. \& Hodkiewicz, M. 2011. The Role of Organizational Factors in Achieving Reliability in the Design and Manufacture of Subsea Equipment. Human Factors and Ergonomics in Manufacturing \& Service Industries, 22(6), 487-505.

da Silva, M.F. \& Radespiel, E.S. 2013. A Diagnostic and Prognostic Framework for Integrated ReservoirCompletion Management Using Intelligent Well Data. Offshore Technology Conference.

Soosaipillai, C., Roald, P.K., Alfstad, D., Aas, T., Smith, G. \& Bressand, J.Y. 2013. Condition Performance monitoring for subsea: experience and value from Gjoa Filed, OTC-24182-MS. Offshore Technology Conference.

Uyiomendo, E. E. \& Markeset, T. 2010. Subsea Maintenance Service Delivery: Mapping Factors Influencing Scheduled Service Duration. Automation and Computing 7(2):167-172.

Uyiomendo, E. E. \& Markeset, T. 2015. Subsea maintenance service delivery: A multi-variable analysis model for predicting potential delays in scheduled services. Quality in Maintenance Engineering 21(1): 34-54.

Vachtsevanos, G., Lewis, F., Roemer, M., Hess, A., \& Wu, B. 2006. Intelligent Fault Diagnosis and Prognosis For Engineering systems. New Jersey: John Wiley. 